\title{
SUBSKY: Efficient Computation of Skylines in Subspaces
}

\author{
Yufei Tao \\ Department of Computer Science \\ City University of Hong Kong \\ Tat Chee Avenue, Hong Kong \\ taoyf@cs.cityu.edu.hk
}

\author{
Xiaokui Xiao \\ Department of Computer Science \\ City University of Hong Kong \\ Tat Chee Avenue, Hong Kong \\ xkxiao@cs.cityu.edu.hk
}

\author{
Jian Pei \\ School of Computing \\ Simon Fraser University \\ University Drive, Burnaby, Canada \\ jpei@cs.sfu.ca
}

\begin{abstract}
Given a set of multi-dimensional points, the skyline contains the best points according to any preference function that is monotone on all axes. In practice, applications that require skyline analysis usually provide numerous candidate attributes, and various users depending on their interests may issue queries regarding different (small) subsets of the dimensions. Formally, given a relation with a large number (e.g., > 10) of attributes, a query aims at finding the skyline in an arbitrary subspace with a low dimensionality (e.g., 2).

The existing algorithms do not support subspace skyline retrieval efficiently because they (i) require scanning the entire database at least once, or (ii) are optimized for one particular subspace but incur significant overhead for other subspaces. In this paper, we propose a technique SUBSKY which settles the problem using a single B-tree, and can be implemented in any relational database. The core of SUBSKY is a transformation that converts multi-dimensional data to $1 D$ values, and enables several effective pruning heuristics. Extensive experiments with real data confirm that SUBSKY outperforms alternative approaches significantly in both efficiency and scalability.
\end{abstract}

\section{Introduction}

Given a set of $d$-dimensional points, a point $p$ dominates another $p^{\prime}$ if the coordinate of $p$ on each dimension is not larger than that of $p^{\prime}$, and strictly smaller on at least one dimension. The skyline consists of all the points that are not dominated by others. Consider, for example, Figure 1 where $d=2$, and each point corresponds to a hotel record. The $\mathrm{x}$-dimension represents the price of a hotel, and the yaxis captures its distance to the beach. Hotel $p_{1}$ dominates $p_{2}$ because the former is cheaper and closer to the beach, meaning that $p_{1}$ is more preferable according to any preference function that is monotone on the two axes [4]. The skyline includes $p_{1}, p_{4}$, and $p_{5}$, which offer various tradeoffs between price and distance: $p_{4}$ is the nearest to the beach, $p_{5}$ is the cheapest, and $p_{1}$ may be a good compromise of the two factors.

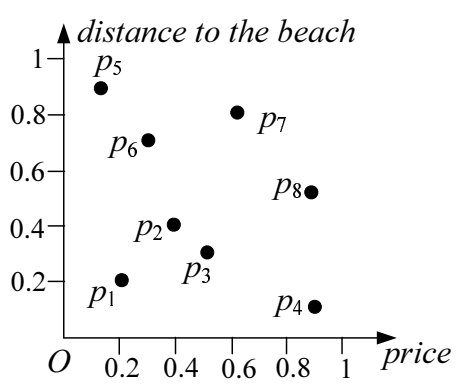

Figure 1. A skyline example

Skyline computation is important in a large number of applications requiring multi-criteria optimization, and has received considerable attention in the database literature $[1,4,5,6,8,9,12,13,14,15,18,19]$. The existing algorithms can be classified in two categories. The first one involves solutions that do not assume any index on the underlying dataset, but they retrieve the skyline by scanning the entire database at least once, resulting in expensive overhead. Methods of the other category incur significantly lower query cost by performing the search on an appropriate structure, e.g., an R-tree [2]. We focus on the second category in this paper.

The motivation of this work is that the previous indexbased approaches are optimized for a fixed set of dimensions, and have poor performance for skyline queries targeting different attributes. For instance, assume that, in addition to the dimensions in Figure 1, the database also records the distances of each hotel to several other locations (e.g., the town center, the nearest supermarket, subway station, etc.), the ratings of security, air-quality, traffic-status in the neighborhood, and so on. In other words, the full data space can have a high dimensionality, but users may want to retrieve the skyline in any subspace containing a small number of axes.

Obviously, a structure constructed in a particular subspace is useless for queries concerning another. To enable skyline retrieval in all subspaces, a straightforward idea is to create an index that covers all the dimensions (e.g., a high-dimensional R-tree). Unfortunately, the previous algorithms suffer considerable performance degradation when 
applied to such a structure (a phenomenon known as "the curse of dimensionality" [3]). Another naive approach is to create multiple low-dimensional structures for different subspaces. For example, assume that the full space has 10 dimensions, and most queries request skylines in subspaces with two attributes. We could create a 2D R-tree for each subspace to achieve the best query efficiency. The number of trees, however, equals $\left(\frac{1}{2}^{10}\right)$, and each coordinate of every point must be duplicated 9 times, leading to expensive space consumption and update overhead.

In fact, applications that require skyline analysis usually provide numerous candidate attributes, and various users may issue queries regarding different (small) subsets of the dimensions depending on their interests. Therefore, a truly practical method for skyline retrieval should aim at effectively supporting queries about subspaces (especially those involving few axes), by consuming the smallest amount of space. In this paper, we present a new technique $S U B S K Y$, which solves the problem using a transformation that converts each $d$-dimensional point to a $1 \mathrm{D}$ value. The converted values are indexed by a single conventional B-tree, which is deployed to find the skyline in any subspace efficiently, based on several interesting observations on the problem characteristics. SUBSKY leverages purely relational technologies, and hence, can be easily implemented in any commercial database system. Extensive experiments with real datasets confirm that $S U B S K Y$ is significantly faster than alternative algorithms.

The rest of the paper is organized as follows. Section 2 reviews the previous work related to ours. Section 3 presents the basic SUBSKY optimized for uniform data, and Section 4 generalizes the technique to arbitrary data distributions. Section 5 contains an extensive experimental evaluation that demonstrates the efficiency of SUBSKY. Section 6 concludes the paper with directions for future work.

\section{Related Work}

The first skyline algorithm in the database context is $B N L$ (block-nested-loop) [4], which compares each point with the others, and reports it as a result only if it is not dominated by any other point. SFS [6] (sort-filter-skyline) is based on the same rationale as $B N L$, but improves performance by first sorting the data according to a monotone function. $D C$ [4] (divide-and-conquer) divides the data space into several regions, calculates the skyline in each region, and produces the final skyline from the points in the regional skylines. Bitmap [18] converts each point $p$ to a bit string, which encodes the number of points having a smaller coordinate than $p$ on every dimension. The skyline is then obtained using only bit operations. LESS (linearelimination-sort for skyline) [9] is an algorithm that has attractive worst-case asymptotical performance. Specifically, when the data distribution is uniform and no two points have

\begin{tabular}{|l|l|l|l|l|}
\hline List 1 (x) & $p_{5}: 0.1$ & $p_{6}: 0.3$ & $p_{2}: 0.4$ & $p_{7}: 0.6$ \\
\hline List 2 (y) & $p_{4}: 0.1$ & $p_{1}: 0.2$ & $p_{3}: 0.3$ & $p_{8}: 0.5$ \\
\hline
\end{tabular}

Figure 2. The structure of Index

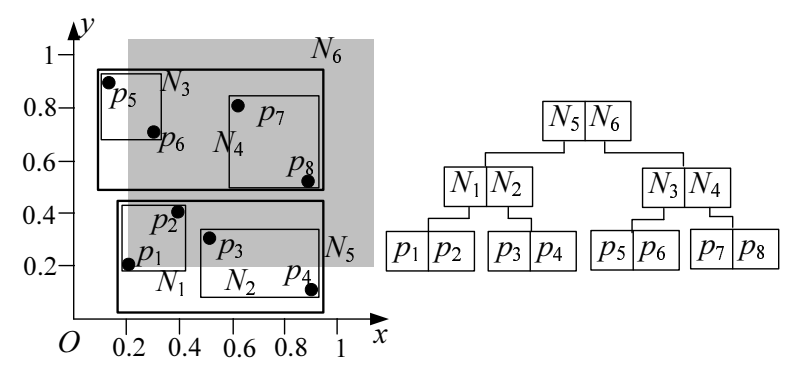

Figure 3. Illustration of $B B S$

the same coordinate on any dimension, LESS computes a $d$ dimensional skyline in expected $O(d \cdot n)$ time, where $n$ is the dataset cardinality.

As opposed to the above algorithms that must read the whole database at least once, index-based methods need to visit only a fraction of the dataset. Index [18] organizes the data into $d$ lists, where the $i$-th list $(1 \leq i \leq d)$ contains points whose coordinates on the $i$-th axis are the smallest among all the dimensions. Figure 2 shows the $d=2$ lists for the dataset of Figure 1. For example, $p_{5}$ is assigned to List 1 because its $\mathrm{x}$-coordinate 0.1 is smaller than its $\mathrm{y}$ coordinate 0.9 . In case a point (e.g., $p_{1}, p_{2}$ ) has identical coordinates on both dimensions, the list that contains it is decided randomly (assume that $p_{2}$ and $p_{1}$ are included in Lists 1 and 2, respectively). The entries in List 1 (2) are sorted in ascending order of their $\mathrm{x}-(\mathrm{y}-)$ coordinates (e.g., entry $p_{5}: 0.1$ indicates the sorting key 0.1 of $p_{5}$ ).

To compute the skyline, Index scans the two lists in a synchronous manner. At the beginning, the algorithm initializes two pointers $p t_{1}$ and $p t_{2}$ referencing the first entries $p_{5}, p_{4}$ of the two lists, respectively. The referenced entry with a smaller sorting key is processed. Since both $p_{5}$ and $p_{4}$ have the same sorting key 0.1 , Index processes the point $p_{5}$ from List 1 , adds it to the skyline set $S_{s k y}$, and moves $p t_{1}$ to the next entry $p_{6}$. The next point examined is $p_{4}$, which is compared with the existing skyline point $p_{5}$, and then inserted in $S_{s k y}$ (i.e., it is not dominated by $p_{5}$ ). Similarly, $p_{1}$ is also included as a skyline point, after which $p t_{2}$ is set to $p_{3}$.

Both coordinates of $p_{1}$ are smaller than the $\mathrm{x}$-coordinate 0.3 of $p_{6}$ (pointed to by $p t_{1}$ ), in which case all the not-yet inspected points $p$ in List 1 can be pruned. To understand this, observe that both coordinates of $p$ are at least 0.3 , indicating that $p$ is dominated by $p_{1}$. Due to the same reasoning, List 2 can also be eliminated because both coordinates of $p_{1}$ are lower than the $\mathrm{y}$-coordinate of $p_{3}$. Thus, the algorithm finishes with $\left\{p_{1}, p_{4}, p_{5}\right\}$ as the result.

$N N$ (nearest-neighbor) [12] and BBS (branch-and-bound 
skyline) [14] find the skyline using an R-tree [2]. The difference is that $N N$ issues multiple $N N$ queries $[17,11]$ while $B B S$ performs only a single traversal of the tree. It has been proved [14] that $B B S$ is I/O optimal, i.e., it accesses fewer disk pages than any algorithm based on R-trees (including $N N$ ). Hence, the following discussion concentrates on this technique. Figure 3 shows the R-tree for the dataset of Figure 1, together with the minimum bounding rectangles (MBR) of the nodes. $B B S$ processes the (leaf/intermediate) entries in ascending order of their mindist (minimum distance) to the origin of the data space. At the beginning, the root entries are inserted into a heap $H\left(=\left\{N_{5}, N_{6}\right\}\right)$ using their mindist as the sorting key. Then, the algorithm removes the top $N_{5}$ of $H$, accesses its child node, and enheaps all the entries there ( $H$ now becomes $\left\{N_{1}, N_{2}, N_{6}\right\}$ ).

Similarly, the next node visited is leaf $N_{1}$, where the data points are also added to $H\left(=\left\{p_{1}, p_{2}, N_{2}, N_{6}\right\}\right)$. Since $p_{1}$ tops $H$, it is taken as the first skyline point, and used for pruning in the subsequent execution. Point $p_{2}$ is de-heaped next, but is discarded because it falls in the dominant region of $p_{1}$ (the shaded area). $B B S$ then visits $N_{2}$, and inserts only $p_{4}$ into $H=\left\{N_{6}, p_{4}\right\}$ ( $p_{3}$ is not inserted as it is dominated by $\left.p_{1}\right)$. Likewise, accessing $N_{6}$ adds only one entry $N_{3}$ to $H\left(=\left\{N_{3}, p_{4}\right\}\right)$ because $N_{4}$ lies completely in the shaded area (i.e., all points in its subtree must be dominated by $p_{1}$ ). The remaining entries processed are $N_{3}$ (en-heaping $p_{5}$ ), $p_{4}, p_{5}$, at which point $H$ becomes empty and $B B S$ terminates.

Finally, Balke et al. [1] consider skyline retrieval in distributed environments, Lin et al. [13] investigate continuous skyline monitoring on data streams, and Chan et al. [5] study partially-ordered domains. These methods are restricted to their specific scenarios, and cannot be adapted for the problem of this paper. Recently, Pei et al. [15] and Yuan et al. [19] independently propose the sky-cube, which consists of the skylines in all possible subspaces. These skylines form a lattice similar to the "data cube" [10] of a relation. The topic of this paper differs from skycube in that we aim at computing the skyline of one particular subspace, as opposed to all subspaces.

\section{The Basic SUBSKY for Uniform Data}

In this section, we focus on uniform data, and explain the rationale of $S U B S K Y$. Section 3.1 presents an algorithm for computing subspace skylines. Then, Section 3.2 provides theoretical evidence that explains its efficiency. In Section 4, we will generalize $S U B S K Y$ to arbitrary data distributions.

\subsection{The Algorithm}

Without loss of generality, we assume a unit $d$ dimensional (full) space where each axis has domain $[0,1]$. We use the term maximal corner for the corner $A^{C}$ of the data space having coordinate 1 on all dimensions. Each data point $p$ is converted to a $1 \mathrm{D}$ value $f(p)$ equal to the $L_{\infty}$ distance between $p$ and $A^{C}$ :

$$
f(p)=\max _{i=1}^{d}(1-p[i])
$$

where $p[i]$ represents the $i$-th coordinate of $p(1 \leq i \leq d)$. If $p_{s k y}$ is a skyline point in the full space, no point $p$ satisfying the following inequality can belong to the skyline:

$$
f(p)<\min _{i=1}^{d}\left(1-p_{s k y}[i]\right)
$$

Figure 4a illustrates a 2D example. Points $p$ satisfying the inequality constitute the shaded square, whose side length equals $\min _{i=1}^{2}\left(1-p_{s k y}[i]\right)$, i.e., the coordinate difference between $p_{s k y}$ and $A^{C}$ on the y-dimension. Obviously, no such $p$ can appear in the skyline because the square is entirely contained in the dominant region of $p_{s k y}$. Consider point $p^{\prime}$ outside the shaded area (i.e., $p^{\prime}$ violates Inequality 2). Given only $f\left(p^{\prime}\right)$ (but not the coordinates of $p^{\prime}$ ), we cannot assert that $p_{s k y}$ dominates $p^{\prime}$ — points whose $L_{\infty}$ distances to $A^{C}$ equal $f\left(p^{\prime}\right)$ could lie anywhere on the left and bottom edges of the dotted square with side length $f\left(p^{\prime}\right)$.

Inequality 2 applies to the original space, while a similar result exists for the skyline of any subspace. Representing a subspace as a set $S U B$ capturing the relevant dimensions (e.g., if the subspace involves the 1 st and 3rd axes of a data space, then $S U B=\{1,3\}$ ), we have:

Lemma 1 Given a skyline point $p_{\text {sky }}$ in a subspace $S U B$, no point $p$ qualifying the following condition can belong to the skyline of $S U B$ :

$$
f(p)<\min _{i \in S U B}\left(1-p_{s k y}[i]\right)
$$

For example, assume that the full space has dimensionality 3 whereas the goal is to retrieve the skyline in the first two dimensions $(S U B=\{1,2\})$. If $p_{s k y}$ has coordinates $(0.05,0.1,-)$ (the 3 rd coordinate is irrelevant), no point $p$ with $f(p)<1-0.1=0.9$ can be in the target skyline. This is correct because the coordinates of $p$ must be at least 0.1 on all dimensions; hence, it is dominated by $p_{s k y}$ in the subspace $S U B$.

Lemma 1 leads to a fast algorithm for computing the skyline of a subspace $S U B$. Specifically, we access the data points $p$ in descending order of their $f(p)$. Meanwhile, we maintain (i) the current set $S_{s k y}$ of skyline points (among the data already examined), and (ii) a value $U$ corresponding to the largest $\min _{i \in S U B}\left(1-p_{s k y}[i]\right)$ (i.e., the right hand side of Inequality 3 ) for the points $p_{s k y} \in S_{s k y}$. The algorithm terminates when $U$ is larger than the $f(p)$ of the next $p$ to be processed.

We illustrate the algorithm using the 8 three-dimensional points of Figure 5. The coordinates of each point $p_{i}(1 \leq$ 


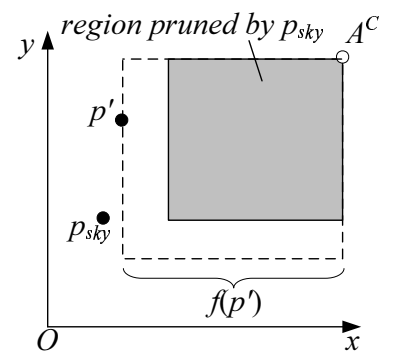

Figure 4. Illustration of Inequality 2

\begin{tabular}{|c|c|c|c|c|c|c|c|c|}
\hline dimension & $p_{1}$ & $p_{2}$ & $p_{3}$ & $p_{4}$ & $p_{5}$ & $p_{6}$ & $p_{7}$ & $p_{8}$ \\
\hline $1(\mathrm{x})$ & 0.2 & 0.4 & 0.5 & 0.9 & 0.1 & 0.3 & 0.6 & 0.9 \\
\hline $2(\mathrm{y})$ & 0.2 & 0.4 & 0.3 & 0.1 & 0.9 & 0.7 & 0.8 & 0.5 \\
\hline $3(\mathrm{z})$ & 0.5 & 0.9 & 0.1 & 0.6 & 0.3 & 0.2 & 0.7 & 0.6 \\
\hline \hline$f\left(p_{i}\right)$ & 0.8 & 0.6 & 0.9 & 0.9 & 0.9 & 0.8 & 0.4 & 0.5 \\
\hline
\end{tabular}

Figure 5. An example dataset

$i \leq 8$ ) correspond to the numbers in the 2 nd-4th rows of the column for $p_{i}$ (the $\mathrm{x}$ - and $\mathrm{y}$-coordinates are the same as in Figure 1). The last row contains the values of $f\left(p_{i}\right)$. Let the query subspace involve the first two dimensions $(S U B=\{1,2\})$. We process the data in this order: $\left\{p_{3}\right.$, $\left.p_{4}, p_{5}, p_{1}, p_{6}, p_{2}, p_{8}, p_{7}\right\}$. After examining $p_{3}$, the algorithm initializes $S_{s k y}$ to $\left\{p_{3}\right\}$, and $U$ to 0.5 (i.e., the smaller between $1-p_{3}[1]$ and $\left.1-p_{3}[2]\right)$. After $p_{4}, S_{s k y}$ becomes $\left\{p_{3}, p_{4}\right\}$, but $U$ remains the same. Similarly, $p_{5}$ is added to $S_{s k y}$ next without affecting $U$. Processing $p_{1}$ inserts it in $S_{s k y}$, removes $p_{3}$ from $S_{s k y}$ ( $p_{3}$ is dominated by $p_{1}$ ), and updates $U$ to 0.8 . Finally, we inspect $p_{6}$, which does not change $S_{s k y}$ or $U$. Since the $f$-values of the remaining points are smaller than the current $U=0.8$, the algorithm finishes and reports $\left\{p_{1}, p_{4}, p_{5}\right\}$ as the final skyline.

In practice, to support dynamic updates, we can index the $f(p)$ of all points $p$ using a B-tree. To achieve the correct access order in answering a skyline query, the algorithm starts with the leaf node containing the largest $f$-values. After exhausting all the entries there, it loads the neighboring leaf (using the "neighbor pointer" in the previous node), and repeats the process until the end of execution.

\subsection{Analysis}

As shown in the experiments, the above solution already significantly outperforms $B B S$ in subspace skyline computation, especially when the dimensionality $d$ of the full space is high, and $B B S$ suffers serious performance degradation. To explain this, consider a 15D uniform dataset with cardinality $100 \mathrm{k}$, and we want to retrieve the skyline in a subspace $S U B$ containing any two dimensions, as shown in Figure 6. There is a high chance that a skyline point lies very close to the origin in $S U B$. Specifically, let us examine the square in Figure 6 whose lower-left corner is the origin, and its side length equals some small $\lambda \in[0,1]$. The

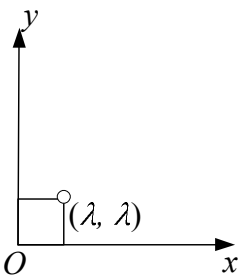

Figure 6. Illustration of the analysis

probability of not having any data point in the square equals $\left(1-\lambda^{2}\right)^{100 k}$, which is less than $10 \%$ for $\lambda=0.001$. In other words, with an at least $90 \%$ chance, we can find a point $p_{s k y}$ in the square such that $\min _{i \in S U B}\left(1-p_{s k y}[i]\right) \geq 0.999$. In this case, (by Inequality 3 ) all points $p$ with $f(p)<0.999$ can be eliminated using the B-tree. The expected percentage of such points in the whole dataset equals the volume of a 15-dimensional square with side length 0.999 (i.e., the square whose opposite corners are the maximal corner of the data space, and the point with coordinate 0.001 on all axes). The volume evaluates to $0.999^{15}=98.5 \%$, that is, we only need to access $1.5 \%$ of the dataset!

\section{The General SUBSKY}

In the previous section (where we concentrated on uniform data), $f(p)$ is always computed with respect to one anchor, i.e., the maximal corner $A^{C}$. In practice where data are usually clustered, the $f(p)$ of various $p$ should be calculated with respect to different anchors to achieve greater pruning power. Section 4.1 elaborates this using concrete examples, and then the subsequent sections develop a new subspace-skyline algorithm based on this idea.

\subsection{Pruning with Multiple Anchors}

Figure 7a shows a 2D dataset, where the data are clustered near the upper-left corner. If $f(p)$ equals the $L_{\infty}$ distance between $p$ and the maximal corner, by Lemma 1 a skyline point $p_{s k y}$ (in the full space) prunes the right shaded square, which, however, is useless since the square does not cover any data point. On the other hand, let $f(p)$ be the $L_{\infty}$ distance from $p$ to an alternative anchor $A_{1}$. We can eliminate all points $p$ whose $f(p)$ is smaller than $\min _{i=1}^{2}\left(A_{1}[i]-p_{s k y}[i]\right)$, which equals the coordinate difference of $p_{s k y}$ and $A_{1}$ on the $\mathrm{y}$-dimension. These points form the left shaded square, which covers a majority of the data, i.e., anchor $A_{1}$ offers much stronger pruning power than $A^{C}$.

Figure $7 \mathrm{~b}$ illustrates another 2D example, where the dataset consists of 4 clusters $S_{1}, S_{2}, \ldots, S_{4}$. SUBSKY would use 3 anchors $A_{1}, A_{2}, A^{C}$, and convert each data point $p$ to a $1 \mathrm{D}$ value $f(p)$ that equals the $L_{\infty}$ distance between $p$ and one assigned anchor dominated by $p$. Assume that $A_{1}$ is the anchor for the points of $S_{1}, A_{2}$ for those of $S_{4}$, and $A^{C}$ for the remaining data. Interestingly, a skyline point 

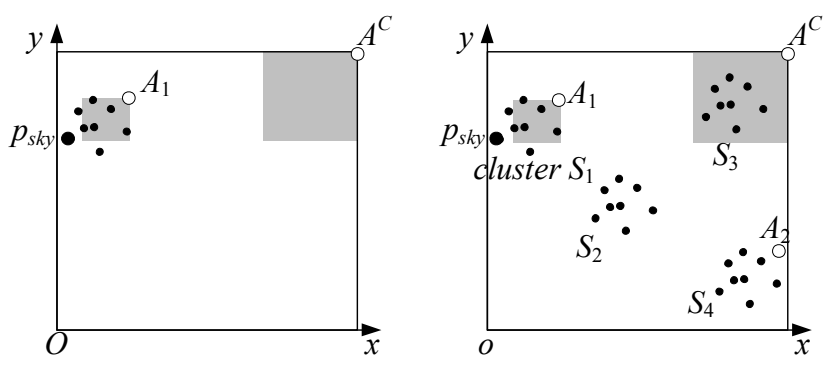

(a) A better anchor $A_{1}$ (b) Anchors for different clusters

Figure 7. Effects of multiple anchors

creates a pruning region with every anchor it dominates. For example, as in Figure $7 \mathrm{a}, p_{s k y}$ from $S_{1}$ can eliminate two shaded squares obtained with $A_{1}$ and $A^{C}$, respectively. In Figure $7 \mathrm{~b}$, the right square includes the entire $S_{3}$, and hence, the data in this cluster can be removed from further consideration.

In general, if the anchor set $S_{a n c}$ contains $A_{1}, A_{2}, \ldots$, $A_{m}$, we have the following heuristic for skyline retrieval in any subspace:

Lemma 2 Let $p_{s k y}$ be a skyline point in a subspace $S U B$, and $S_{\text {anc }}^{\prime}$ the set of anchors whose projections in $S U B$ are dominated by $p_{\text {sky }}$. Then, for each anchor $A \in S_{a n c}^{\prime}$, a data point $p$ assigned to $A$ cannot be in the skyline if

$$
f(p)<\min _{i \in S U B}\left(A[i]-p_{s k y}[i]\right)
$$

The lemma degenerates into Lemma 1 for $A=A^{C}$ (i.e., $A[i]=1)$. As another example, assume $d=3$, and an anchor $A=(0.8,0.7,0.1)$. In the subspace $S U B=\{1,2\}$, a skyline point $p_{s k y}=(0.2,0.2,-)$ eliminates all points $p$ assigned to $A$ with $f(p)<0.5\left(=A[2]-p_{s k y}[2]\right)$. Note that the first and second coordinates of $p$ must be larger than 0.3 and 0.2 respectively, indicating that $p$ is dominated by $p_{s k y}$ in $S U B$. In the original 3D space, however, a skyline point $p_{s k y}=(0.2,0.2,0.2)$ does not produce any pruning region with respect to $A$, since $p_{s k y}$ does not dominate $A$ in this case.

The effectiveness of the heuristic depends on (i) how the data are assigned to anchors, and (ii) how the anchors are selected. We analyze these issues in the next two sections.

\subsection{Assigning Points to Anchors}

We introduce the concept of effective region (ER), which quantifies the benefit of assigning a point to an anchor. Consider Figure 8a where point $p$ is assigned to $A^{C}$ (the maximal corner). Observe that, in computing the skyline in the $2 \mathrm{D}$ space, $p$ can be eliminated with Lemma 2 only if a point is discovered in the shaded square cornered at the origin with side length $1-f(p)$. The square is the ER of $p$, which contains the points that (i) dominate $p$, and (ii) their pruning regions with respect to $A^{C}$ cover $p$ (the coordinate of

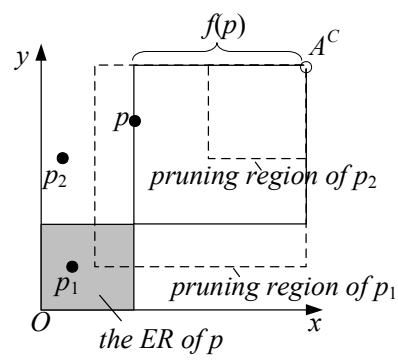

(a) Assigning $p$ to $A^{C}$

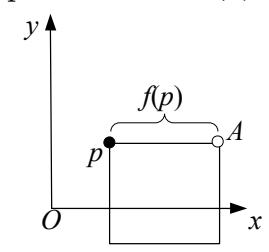

(c) ER is empty

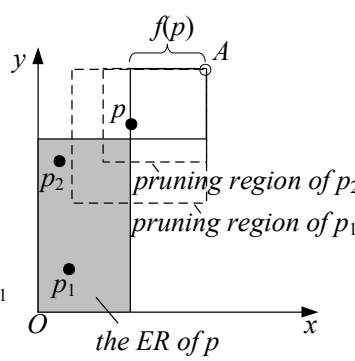

(b) Assigning $p$ to $A$

\section{Figure 8. The concept of effective region}

any point in the ER differs from that of $A^{C}$ by more than $f(p)$ on all axes). For example, since $p_{1}\left(p_{2}\right)$ is inside (outside) the ER, the pruning region of $p_{1}\left(p_{2}\right)$ contains (does not contain) $p$.

Let us assign $p$ to an alternative anchor $A$ in Figure $8 \mathrm{~b}$. The shaded area demonstrates the new ER of $p$, and it covers both $p_{1}$ and $p_{2}$ (the dashed rectangles are their pruning regions). This means that $p$ can be eliminated as long as either $p_{1}$ or $p_{2}$ has been discovered. Compared with assigning $p$ to $A^{C}$, the new assignment increases the chance of pruning $p$.

In general, given a point $p$ and an anchor $A$ dominated by $p$, the ER of $p$ (with respect to $A$ ) is a d-dimensional rectangle whose opposite corners are the origin and the point having coordinate $A[i]-L_{\infty}(p, A)$ on the $i$-th dimension $(1 \leq i \leq d)$, where $L_{\infty}(p, A)$ is the $L_{\infty}$ distance between $p$ and $A$, and also the value of $f(p)$ if $p$ is assigned to $A$. The ER is empty if $A[i]<L_{\infty}(p, A)$ for any dimension $i$. Figure $8 \mathrm{c}$ shows such an example $(d=2)$, where the y-coordinate of $A$ is smaller than $L_{\infty}(p, A)$, indicating that there does not exist any skyline point that can prune $p$ using Lemma 2.

Hence, we assign $p$ to the anchor that produces the largest ER. Specifically, this is the anchor that is dominated by $A$ and maximizes:

$$
\Pi_{i=1}^{d} \max \left(0, A[i]-L_{\infty}(p, A)\right)
$$

What is the connection between ER (formulated in the full space) and skyline retrieval in a subspace? To answer this question, we need to interpret Lemma 2 in an alternative manner. Given a skyline point $p_{s k y}$ in the query subspace $S U B$, let us obtain a point $p_{s k y}^{\prime}$ in the original space whose coordinates are equal to those of $p_{s k y}$ on the dimensions included in $S U B$, and 0 on the other axes. Then, a point 


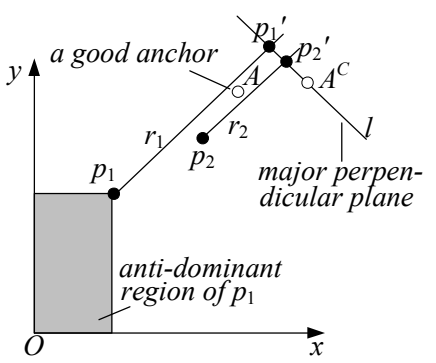

(a) Perfect rays

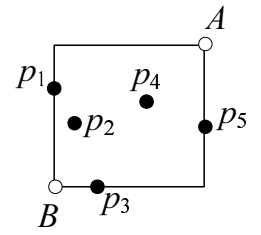

(b) Deciding the anchor
Figure 9. Finding anchors

$p$ can be pruned by Lemma 2 (i.e., $p$ does not belong to the skyline of $S U B$ ) if and only if $p_{s k y}^{\prime}$ lies in the ER of $p$. Therefore, enlarging ER also increases the probability of pruning $p$ in computing a subspace skyline.

\subsection{Finding the Anchors}

An anchor that leads to a large ER for one data point may produce a small ER for another. When we are allowed to keep only $m$ anchors (where $m$ is a small system parameter), how should they be selected in order to maximize the ER volumes of as many points as possible?

Note that the largest ER of a point $p$ corresponds to its anti-dominant region, consisting of all the points dominating $p$. In other words, the maximum value of Formula 5 equals $\prod_{i=1}^{d} p[i]$, which is achieved when

$$
A[i]-L_{\infty}(p, A)=p[i]
$$

on all dimensions $i \in[1, d]$. We call an anchor $A$ satisfying the above equation the perfect anchor for $p$. If $p$ is assigned to $A$, then $p$ can be pruned with Lemma 2 using any skyline point dominating $p$.

It turns out that each point $p$ has infinite perfect anchors. Let us shoot a ray from $p$ that is in its dominant region, and parallel to the major diagonal of the data space (i.e., the diagonal connecting the origin and the maximal corner); every point $A$ on this perfect ray is a perfect anchor of $p$ (even including the part of the ray out of the data space). In fact, the coordinate difference between $A$ and $p$ is equivalent on all axes, i.e., $A[i]-p[i]=L_{\infty}(p, A)$ for any $i \in[1, d]$, leading to the correctness of Equation 6 .

In Figure 9a, for example, the perfect ray of point $p_{1}$ is $r_{1}$, and any anchor on $r_{1}$ will result in the ER of $p_{1}$ that is the shaded rectangle. Similarly, $r_{2}$ is the ray for $p_{2}$. Since $r_{1}$ and $r_{2}$ are very close to each other, if we can keep only a single anchor $A$, it would lie between the two rays as in Figure $9 \mathrm{a}$ - although $A$ is not the perfect anchor of $p_{1}$ and $p_{2}$, it is a good anchor as it leads to large ERs for both points.

The important implication of the above discussion is that points with close perfect rays may share the same anchor. This observation naturally leads to an algorithm for finding anchors based on clustering. Specifically, we first project

\begin{tabular}{|l|c|c|c|c|c|c|c|c|}
\hline & $p_{1}$ & $p_{2}$ & $p_{3}$ & $p_{4}$ & $p_{5}$ & $p_{6}$ & $p_{7}$ & $p_{8}$ \\
\hline ER-vol w.r.t. $A_{1}$ & 8 & 64 & 1 & 1 & 1 & 8 & 216 & 125 \\
\hline ER-vol w.r.t. $A_{2}$ & 0 & - & 9 & - & - & 9 & 144 & 75 \\
\hline
\end{tabular}

(a) ER volumes with respect to $A_{1}, A_{2}$ (unit $10^{-3}$ )

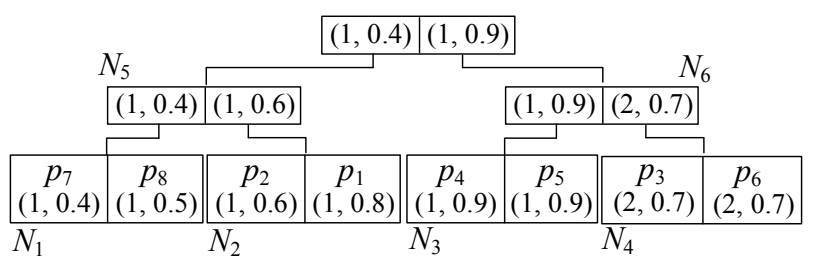

(b) The B-tree on the transformed $f$-values

\section{Figure 10. Illustration of the skyline algorithm}

the data onto the major perpendicular plane, which is the $d$-dimensional plane (passing the maximal corner) perpendicular to the major diagonal of the data space. In Figure 9a $(d=2)$, for instance, the plane is line $l$, and the projections of $p_{1}$ and $p_{2}$ are $p_{1}^{\prime}$ and $p_{2}^{\prime}$, respectively. Then, we partition the projected points into $m$ clusters using the k-means algorithm [16, 7], and then formulate an anchor for each cluster. Consider again Figure 7b, where the data in $S_{2}$ and $S_{3}$ collapse into a single cluster in the major perpendicular plane, which explains why these points were assigned to the same anchor.

It remains to clarify how to decide an anchor $A$ for a cluster $S$. We aim at guaranteeing that $A$ should produce a non-empty ER for every point $p \in S$ (i.e., $A[i]>L_{\infty}(p, A)$ on every dimension $i$, as discussed in Section 4.2); otherwise, $p$ cannot be assigned to $A$. We illustrate the algorithm using a $2 \mathrm{D}$ example but the idea generalizes to arbitrary dimensionality in a straightforward manner.

Assume that $S$ consists of 5 points, and the algorithm examines them in the original space (i.e., not their projections), as shown in Figure 9b. We first obtain point $B$, whose coordinate on each dimension equals the lowest coordinate of the points in $S$ on this axis (note that $B$ necessarily falls inside the data space, and dominates all the points). Then, the algorithm computes the smallest square (or a hyper-square in $d$-dimensional space) that covers all the points (see Figure 9b). The anchor $A$ for $S$ is the corner of the square opposite to $B$. The pruning region of $B$ with respect to $A$ encloses the entire square, or equivalently, the ER of every point is not empty since it includes at least $B$.

\subsection{The Data Structure and Query Algorithm}

Given a small number $m$ (less than 100 in our experiments), SUBSKY first obtains $m$ anchors, by applying the method of Section 4.3 on a random subset of the database. Then, the $f(p)$ of each point $p$ is set to the $L_{\infty}$ distance between $p$ and its assigned anchor (which maximizes the vol- 
Algorithm subsky ( $\left.S U B,\left\{A_{1}, A_{2}, \ldots A_{m}\right\}\right)$

/* $S U B$ includes the dimensions relevant to the query

subspace; $A_{1}, \ldots, A_{m}$ are the anchors */

1. for $j=1$ to $m$

2. use the B-tree to find the point $p t_{j}$ with the maximum $f\left(p t_{j}\right)$ among all the points assigned to $A_{j}$

3. $p t_{j} . E R=\Pi_{i=1}^{d}\left(A_{j}[i]-L_{\infty}\left(p t_{j}, A_{j}\right)\right)$ //Equation 5

4. $S_{s k y}=\emptyset / /$ the set of skyline points

5. while $\left(p t_{j} \neq \emptyset\right.$ for any $\left.j \in[1, m]\right)$

6. $t=$ the value of $j$ giving the smallest $p t_{j}$.ER among all $j \in[1, m]$ such that $p t_{j} \neq \emptyset$

7. if $p t_{t}$ is not dominated by any point in $S_{s k y}$

8. remove from $S_{s k y}$ the points dominated by $p t_{t}$

9. $S_{s k y}=S_{s k y} \cup\left\{p t_{t}\right\}$

10. for $j=1$ to $m$

11. if $p t_{j} \neq \emptyset$ and $f\left(p t_{j}\right)<\min _{i \in S U B}\left(A_{j}[i]-p t_{t}[i]\right)$

12. $p t_{j}=\emptyset / *$ no point assigned to $A_{j}$ can belong to the skyline (Lemma 2) */

13. $p t_{t}=$ the point with the next largest $f(p)$ among the data assigned to $A_{t}$ (this point lies in either the same leaf as the previous $p t_{t}$, or a neighboring node)

14. if no more such point exists then $p t_{t}=\emptyset$

15. else $p t_{t} . E R=\Pi_{i=1}^{d}\left(A_{t}[i]-L_{\infty}\left(p t_{t}, A_{t}\right)\right)$

16. return $S_{s k y}$

\section{Figure 11. The query algorithm of $S U B S K Y$}

ume of ER among the anchors dominated by $p^{1}$ ). We guarantee the existence of such an anchor by always including the maximal corner in the anchor set.

SUBSKY manages the resulting $f(p)$ with a single B-tree that separates the points assigned to various anchors. We achieve this by indexing a composite key $(j, f(p))$, where $j \in[1, m]$ is the id of the anchor to which $p$ is assigned. Thus, an intermediate entry $e$ of the B-tree has the form (e.id, e.f), which means that (i) each point $p$ in the subtree of $e$ has been assigned to the $j$-th anchor with $j \geq e . i d$, and (ii) in case $j=e . i d$, the value of $f(p)$ is at least $e . f$.

We illustrate the above process using the $3 \mathrm{D}$ dataset of Figure 5 and $m=2$ anchors: the maximal corner $A_{1}$, and $A_{2}=(1,1,0.8)$. The second row of Figure 10a illustrates the ER volume of each data point with respect to $A_{1}$, calculated by Equation 5. For instance, the volume 0.125 of $p_{8}$ is $\Pi_{i=1}^{3}\left(A_{1}[i]-L_{\infty}\left(A_{1}, p_{8}\right)\right)=(1-0.5)^{3}$. Similarly, the third row contains the ER volumes with respect to $A_{2}$. A "-" means that the corresponding ER does not exist. For example, the ER of $p_{2}$ is undefined because $p_{2}$ does not dominate $A_{2}$, while there is no ER for $p_{4}$ since $A_{2}[3]=0.8$ is smaller than $L_{\infty}\left(A_{2}, p_{4}\right)=0.9$ (review the formulation of ER in Section 4.2). The white cells of the table indicate each point's ER-volume with respect to its assigned anchor. For example, $p_{3}$ is assigned to $A_{2}$ since this anchor produces a

\footnotetext{
${ }^{1}$ The approach is better than assigning $p$ to its closest anchor in the major perpendicular plane, because the ER-volume directly quantifies the benefit of an assignment.
}

larger ER than $A_{1}$. Figure 10b shows the B-tree indexing the transformed $f$-values, e.g., the leaf entry $p_{3}:(2,0.7)$ in node $N_{4}$ captures the fact that $f\left(p_{3}\right)$ equals the $L_{\infty}$ distance 0.7 between $A_{2}$ and $p_{3}$.

Assume that we aim at computing the skyline in the subspace $S U B=\{1,2\}$. As the first step, the algorithm identifies, for each anchor, the assigned data point $p$ with the maximum $f(p)$. In Figure $10 \mathrm{~b}$, the point for $A_{1}\left(A_{2}\right)$ is $p_{6}$ $\left(p_{5}\right)$, which is the right-most point assigned to this anchor at the leaf level, and can be easily found by accessing a single path of the B-tree.

Then, the algorithm scans the points assigned to each anchor in descending order of their $f$-values, i.e., the ordering is $\left\{p_{5}, p_{4}, p_{1}, p_{2}, p_{8}, p_{7}\right\}$ for $A_{1}$, and $\left\{p_{6}, p_{3}\right\}$ for $A_{2}$. For this purpose, we initialize two pointers $p t_{1}$ and $p t_{2}$ referencing the heads $p_{5}$ and $p_{6}$ of the two lists, respectively. At each iteration, we process the referenced point with a smaller ER (in case of tie, the next processed point is randomly decided). Continuing the example, since the ERvolume 1 of $p_{5}$ is smaller than that 9 of $p_{6}$ (implying that $p_{6}$ has a larger probability of being pruned by a future skyline point), the algorithm adds $p_{5}$ to the skyline set $S_{s k y}$, and advances $p t_{1}$ to the next point $p_{4}$ in the list of $A_{1}$. Similarly, $p_{4}$ has a lower ER-volume 1 (than $p_{6}$ ), and is not dominated by $p_{5}$; thus, it is also added to $S_{s k y}\left(=\left\{p_{5}, p_{4}\right\}\right)$. Pointer $p t_{1}$ now reaches $p_{1}$ (with ER volume 1), which is included in $S_{s k y}$, too.

According to Lemma $2, p_{1}$ prunes all the points $p$ assigned to $A_{1}$ whose $f(p)$ are smaller than $\min _{i \in S U B}\left(A_{1}[i]-p_{1}[i]\right)=0.8$. Since the next point $p_{2}$ in the list of $A_{1}$ qualifies the condition, none of the remaining data in the list can be a skyline point. Similarly, $p_{1}$ also prunes the data $p$ assigned to $A_{2}$ satisfying $f(p)<\min _{i \in S U B}\left(A_{2}[i]-p[i]\right)=0.8$. Thus, the head $p_{6}$ in the list of $A_{2}$ is also eliminated $\left(f\left(p_{6}\right)=0.7<0.8\right)$, and no point in the list belongs to the skyline either. Hence, the algorithm terminates with $S_{s k y}\left(=\left\{p_{5}, p_{4}, p_{1}\right\}\right)$. Recall that the basic SUBSKY of Section 3.1 needs to inspect additional data $p_{6}$ and $p_{3}$. Figure 11 formally describes the general algorithm for retrieving a subspace skyline.

\subsection{Discussion}

We keep the anchor set in memory since it is small (occupying only several k-bytes) and is needed for performing queries and updates. Specifically, to insert/delete a point $p$, we decide its assigned anchor $A$ as described in Section 4.2, and set $f(p)=L_{\infty}(p, A)$, after which the insertion/deletion proceeds as in a normal B-tree. Note that the anchor set is never modified after its initial computation. Query efficiency remains unaffected as long as the data distribution does not incur significant changes (we will verify this later with experiments).

For a dynamic dataset, all the data must be retained because a non-skyline point may appear in the skyline after a 


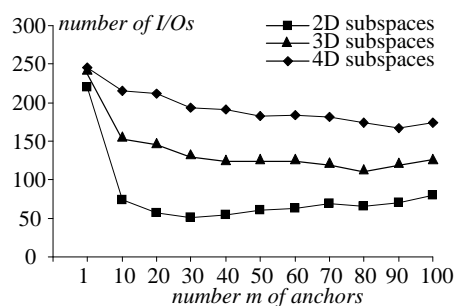

(a) $N B A$

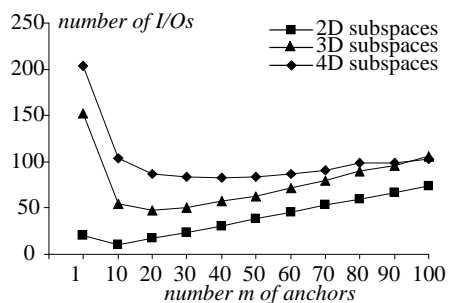

(b) Household

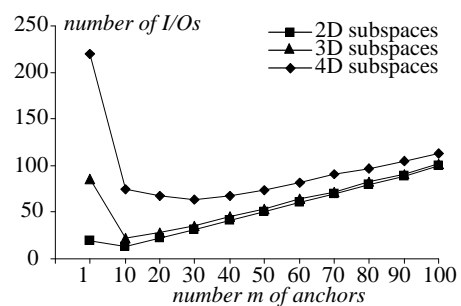

(c) Color

Figure 12. $S U B S K Y$ performance vs. the number of anchors

skyline point is deleted. On the other hand, if the dataset is static, points that are not in the skyline of the full space can be discarded since they will not appear in the skyline of any subspace ${ }^{2}$. When $d$ is large, the size of the full-space skyline may still be comparable to the dataset cardinality [8]. Hence, the points (of the skyline) should be managed by a disk-oriented technique (such as $S U B S K Y$ ) to enable efficient retrieval in subspaces.

So far our definition of "dominance" prefers small coordinates on all dimensions, whereas in general a point may be considered dominating another only if its coordinates are larger on some axes. For example, given two attributes price and size of house records, a reasonable skyline would seek to minimize the price but maximize the size (i.e., a customer is typically interested in large houses with low prices). Depending on its semantics, a dimension usually has only one "preference direction", e.g., skylines involving price (size) would most likely prefer the negative (positive) direction of this axis. SUBSKY easily supports a positive preference direction by subtracting (from 1) all coordinates on the corresponding dimension (e.g., $1-$ price).

\section{Experiments}

In this section, we experimentally evaluate the efficiency of the proposed techniques. We deploy 3 real datasets $N B A$, Household, and Color ${ }^{3}$. Specifically, NBA contains 17k 13dimensional points, where each point corresponds to the statistics of a player in 13 categories including the number of points scored, rebounds, assists etc. averaged over the number of minutes played. Household consists of $127 \mathrm{k}$ 6-dimensional tuples, each of which represents the percentage of an American family's annual income spent on 6 types of expenditures (gas, electricity, etc.). Color is a 9dimensional dataset with a cardinality $68 \mathrm{k}$, and each tuple captures several properties of an image such as brightness, contrast, saturation, and so on. All the values are normalized into the unit range $[0,1]$.

\footnotetext{
${ }^{2}$ Strictly speaking, this is correct only if all the data points have distinct coordinates on each dimension. If this is not true, the points that need to be retained include those sharing common coordinates with a point in the full-space skyline. Retrieval of such points is discussed in [15].

${ }^{3}$ These datasets can be downloaded at http://www.nba.com, http://www.ipums.org, and http://kdd.ics.uci.edu, respectively.
}

We also generate synthetic data with two distributions. A uniform dataset includes random points in a unit space. To create a clustered dataset with cardinality $N$, we first pick 10 cluster centroids randomly. Then, for each centroid, we obtain $N / 10$ points whose coordinate on each axis follows a Gaussian distribution with variance 0.05 , and a mean equal to the corresponding coordinate of the centroid.

Each workload contains 100 queries that request the skylines of 100 random subspaces with the same dimensionality $d_{s u b}$. For example, for $N B A$ and $d_{s u b}=3$, each of the $\left(\begin{array}{l}13 \\ 3\end{array}\right)$ three-dimensional subspaces has an equal probability of being queried. If the number of possible subspaces is smaller than 100 (e.g., for Color, the number of 3D subspaces equals $\left(\begin{array}{l}6 \\ 3\end{array}\right)=20$ ), all the subspaces are inspected. For NBA and Household, each skyline aims at maximizing the coordinates of the participating dimensions, while queries on the other datasets prefer small coordinates.

We compare $S U B S K Y$ against $B B S$ which is the best existing method for skyline computation (see Section 2). Each dataset is indexed by a B-tree (for $S U B S K Y$ ) and an R-tree (for $B B S$ ), where the page size is set to $4 \mathrm{k}$ bytes in all cases. Each B-tree is constructed with anchors computed (as described in Section 4.3) from a 10\% random sample set of the underlying dataset.

Tuning the Number of Anchors. The first set of experiments examines the influence of the number $m$ of anchors on the performance of SUBSKY. For each real dataset, we create 11 B-trees by varying $m$ from 1 to 100 ; then, we use each tree to process a workload, and measure the average (per-query) number of page accesses. Figure 12 plots the cost as a function of $m$, for workloads with $d_{s u b}=2,3$ and 4 , respectively. Note that the result for $m=1$ corresponds to the overhead of the basic SUBSKY that uses the maximal corner as the only anchor (Section 3).

As $m$ becomes larger, the query overhead first decreases and then actually increases after $m$ passes certain threshold. The initial decrease confirms the analysis of Section 4 that query efficiency can be improved by using multiple anchors. To explain the performance deterioration, recall that the query algorithm of $S U B S K Y$ essentially scans $m$ segments of continuous leaf nodes in the B-tree, which require at least $m$ page accesses. For excessively large $m$, these $m$ accesses constitute a dominant factor in the overall 


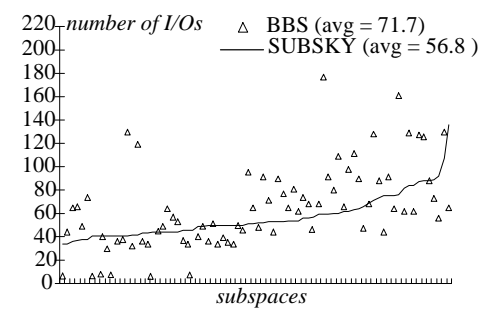

(a) $N B A, d_{s u b}=2$

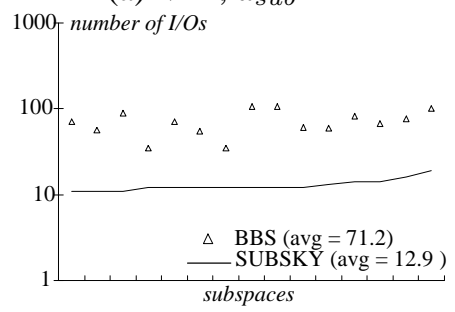

(d) Household, $d_{s u b}=2$

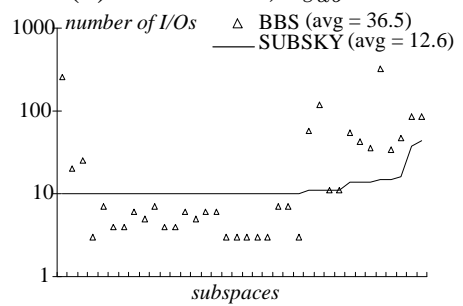

(g) Color, $d_{\text {sub }}=2$

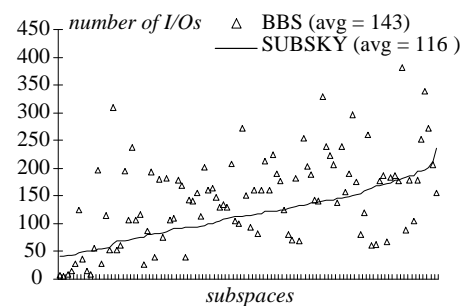

(b) $N B A, d_{s u b}=3$

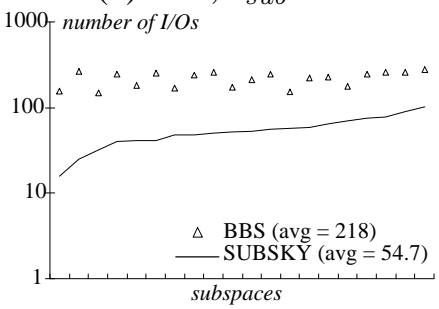

(e) Household, $d_{\text {sub }}=3$

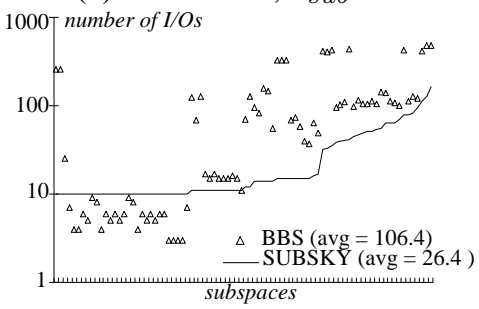

(h) Color, $d_{s u b}=3$

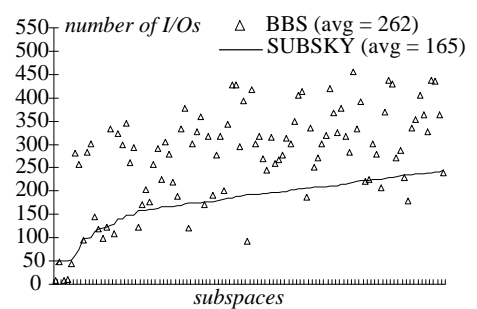

(c) $N B A, d_{s u b}=4$

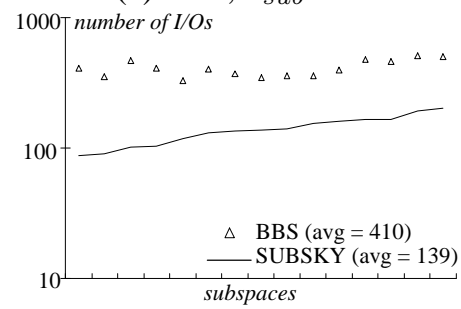

(f) Household, $d_{s u b}=4$

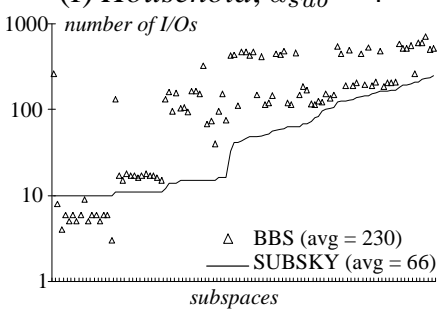

(i) Color, $d_{\text {sub }}=4$

Figure 13. $S U B S K Y$ vs. $B B S$ (real datasets)

overhead, which thus grows (almost) linearly with $m$ (for $N B A$ and $d_{s u b} \geq 2$, the linear phenomenon happens after $m \geq 90$ ).

Even for the same dataset, the optimal $m$ depends on the dimensionality of the subspace queried. Furthermore, the optimal value for a higher $d_{s u b}$ is greater than that for a lower $d_{\text {sub }}$ (e.g., for $N B A$, the best $m$ equals $30,80,90$ for $d_{s u b}=2,3$, and 4, respectively). This is expected because the number of clusters is larger in a higher-dimensional subspace, as the projection of two clusters onto a lowerdimensional subspace may collapse into a single cluster. In the sequel, we set $m$ to 50, 20, 10 for NBA, Household, and Color which result in the best overall performance for the $d_{\text {sub }}$ values tested.

Examination of Individual Subspaces. Figure 13a illustrates the cost of $S U B S K Y$ and $B B S$ for answering each query (in the $2 \mathrm{D}$ workload) on the $N B A$ dataset. The x-axis represents the subspaces, sorted in ascending order of the corresponding SUBSKY overhead. The average cost of each method is shown after its legend (e.g., the per-query overhead of $S U B S K Y$ equals $71.7 \mathrm{I} / \mathrm{Os}$ ). In Figures $13 \mathrm{~b}$ and $13 \mathrm{c}$, we demonstrate a similar comparison for workloads with $d_{\text {sub }}=3$ and 4 , respectively. Figures13d-13i present the results of the same experiments on Household and Color respectively, except that the y-axes are in logarithmic scale.

SUBSKY consistently achieves lower average cost than

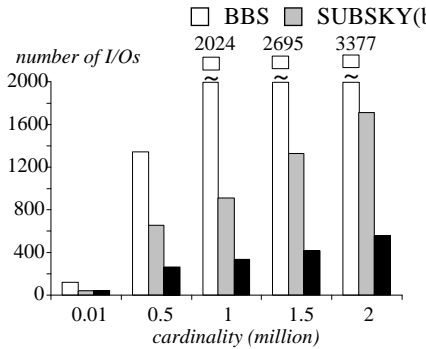

(a) Uniform
SUBSKY

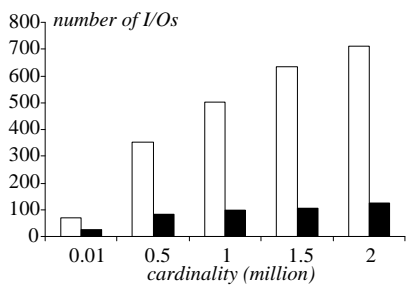

(b) Clustered
Figure 14. Cost vs. cardinality (3D subspaces, 10D full-space)

its competitor (with the maximum speedup 5 in Figure 13d). Regarding individual query performance, $S U B S K Y$ outperforms $B B S$ in all queries on Household, and most queries on $N B A$ and Color. The only exception is in Figure $13 \mathrm{~g}$, where $B B S$ is slightly faster (by less than $5 \mathrm{I} / \mathrm{Os}$ ) for around $60 \%$ of the workload, but significantly slower for the remaining queries, rendering its average overhead 3 times higher than that of SUBSKY.

Scalability with the Cardinality and Full-Space Dimensionality. Next, we deploy 10D uniform datasets with cardinalities ranging from 10 thousand to 2 million. For each dataset, we decide (for $S U B S K Y$ ) the best number $m$ of anchors that optimize the overall performance for 2-4D subspaces (through a tuning process similar to Figure 12). 


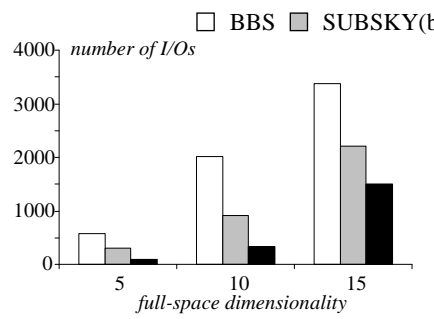

(a) Uniform

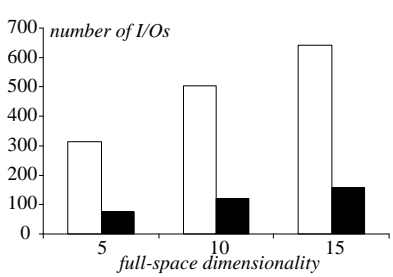

(b) Clustered
Figure 15. Cost vs. dataset dimensionality (3D subspaces, 1 million cardinality)

Specifically, the best value equals 20 for the dataset containing 10k points, and 70 for the others. Figure 14a compares the average cost of $B B S$ and the two versions of $S U B S K Y$ in executing 3D workloads. Evidently, the proposed methods scale better with cardinality than $B B S$. In particular, for cardinality 2 million, $S U B S K Y$ outperforms $B B S$ by almost an order of magnitude. Figure 14b illustrates the corresponding results for Clustered data, confirming similar observations (the $m$ of $S U B S K Y$ equals 10 for the smallest dataset, and 30 for the other cardinalities). The basic SUBSKY is omitted because it targets specifically uniform data.

To examine the influence of the full-space dimensionality $d_{\text {full }}$, we use datasets with cardinality 1 million whose $d_{\text {full }}$ varies from 5 to 15. In Figure 15, we measure the cost of each method (in retrieving skylines of 3D subspaces) as a function of $d_{f u l l}$, for uniform and clustered distributions. $S U B S K Y$ again outperforms $B B S$ significantly.

\section{Conclusions}

In practice, skyline queries are usually issued in a large number of subspaces, each of which includes a small subset of the attributes in the underlying relation. In this paper, we develop a new technique SUBSKY that supports subspace skyline retrieval with only relational technologies. The core of $S U B S K Y$ is a transformation that converts multidimensional data to $1 \mathrm{D}$ values, and permits indexing the dataset with a single conventional B-tree. Extensive experiments verify that $S U B S K Y$ consistently outperforms the previous solutions in terms of efficiency and scalability.

This work also lays down a foundation for future investigation of several related topics. For instance, certain attributes in the relation may appear in the subspaces of most queries (e.g., a user looking for a good hotel would always be interested in the price dimension). In this case, the data structure may be modified to facilitate pruning on these axes. Another interesting issue is to cope with datasets where the data distribution may incur frequent changes. Instead of periodically reconstructing the B-tree, a better approach is to replace only some anchors, and re-organize the data assigned to them. This strategy achieves lower update cost since it avoids accessing the points assigned to the unaffected anchors.

\section{Acknowledgements}

Yufei Tao and Xiaokui Xiao were supported by RGC Grant CityU 1163/04E from the HKSAR government. Jian Pei was supported by an NSERC Discovery Grant and NSF Grant IIS-0308001.

\section{References}

[1] W.-T. Balke, U. Guntzer, and J. X. Zheng. Efficient distributed skylining for web information systems. In $E D B T$, pages 256-273, 2004.

[2] N. Beckmann, H.-P. Kriegel, R. Schneider, and B. Seeger. The $\mathrm{R}^{*}$-tree: An efficient and robust access method for points and rectangles. In SIGMOD, pages 322-331, 1990.

[3] S. Berchtold, D. A. Keim, and H.-P. Kriegel. The X-tree : An index structure for high-dimensional data. In $V L D B$, pages 28-39, 1996.

[4] S. Borzsonyi, D. Kossmann, and K. Stocker. The skyline operator. In ICDE, pages 421-430, 2001

[5] C.-Y. Chan, P.-K. Eng, and K.-L. Tan. Stratified computation of skylines with partially-ordered domains. In SIGMOD, pages 203-214, 2005.

[6] J. Chomicki, P. Godfrey, J. Gryz, and D. Liang. Skyline with presorting. In ICDE, pages 717-719, 2003.

[7] R. O. Duda and P. E. Hart. Pattern Classification and Scene Analysis. Wiley, 1973.

[8] P. Godfrey. Skyline cardinality for relational processing. In FoIKS, pages 78-97, 2004.

[9] P. Godfrey, R. Shipley, and J. Gryz. Maximal vector computation in large data sets. In $V L D B$, pages 229-240, 2005.

[10] J. Gray, A. Bosworth, A. Layman, and H. Pirahesh. Data cube: A relational aggregation operator generalizing groupby, cross-tab, and sub-total. In ICDE, pages 152-159, 1996.

[11] G. R. Hjaltason and H. Samet. Distance browsing in spatial databases. TODS, 24(2):265-318, 1999.

[12] D. Kossmann, F. Ramsak, and S. Rost. Shooting stars in the sky: An online algorithm for skyline queries. In $V L D B$, pages 275-286, 2002.

[13] X. Lin, Y. Yuan, W. Wang, and H. Lu. Stabbing the sky: Efficient skyline computation over sliding windows. In ICDE, 2005.

[14] D. Papadias, Y. Tao, G. Fu, and B. Seeger. Progressive skyline computation in database systems. TODS, 30(1):41-82, 2005.

[15] J. Pei, W. Jin, M. Ester, and Y. Tao. Catching the best views of skyline: a semantic approach based on decisive subspaces. In $V L D B$, pages 253-264. VLDB Endowment, 2005.

[16] D. Pelleg and A. W. Moore. X-means: Extending k-means with efficient estimation of the number of clusters. In International Conference on Machine Learning, pages 727-734, 2000.

[17] N. Roussopoulos, S. Kelley, and F. Vincent. Nearest neighbor queries. In SIGMOD, pages 71-79, 1995.

[18] K.-L. Tan, P.-K. Eng, and B. C. Ooi. Efficient progressive skyline computation. In $V L D B$, pages 301-310, 2001.

[19] Y. Yuan, X. Lin, Q. Liu, W. Wang, J. X. Yu, and Q. Zhang. Efficient computation of the skyline cube. In $V L D B$, pages 241-252, 2005. 